\title{
Multilingual and Multimodal Repertoires as part of Identity Management on Facebook: A Case of International Students
}

\author{
Osman Solmaz \\ Dicle University, Turkey
}

\begin{abstract}
The present study examines the multilingual and multimodal repertoires of international graduate students on Facebook, displaying how semiotic and linguistic resources are employed to index multiple aspects of their identity in social media. Drawing on data from semistructured interviews and social media data of participants, this paper reveals that multilingual students frequently engage in linguistic and multimodal practices by mobilizing a great diversity of semiotic resources in their networks. The findings highlight that international students effectively use their repertoires to deal with a multitude of audiences and build identification performances signaling local and global affiliations. In this respect, the study contributes to the current thinking and investigation of identity management in social media through a mixed-methods approach including social network analysis.
\end{abstract}

Keywords: Facebook, identity, international students, social media

Internet users around the world have access to an enormous amount of information and engage in interactions with others in online spaces. Social media sites are among the spaces that bring spatially removed people of various cultures and backgrounds together in an unbounded context. The number of people engaging in such participatory contexts is growing fast. For instance, Facebook, one of the most popular social networking sites (SNSs) in the world, has 2.07 billion active users per month (Facebook, 2017).

Beyond value as communicative mediums, SNSs like Facebook are prime examples of digitally-mediated spaces where people take part in "Participatory Culture" (Jenkins, 2006), and they are consumers and producers of the content. Individuals create, entextualize, and share content within their networks, with almost no barrier for civic engagement and artistic expression. Opportunities for hybrid and multilingual practices are available in these spaces where content can be converged, and multiple media resources can be used in the efficient involvement in the participatory culture. Users take advantage of a range of affordances for "discourse practice, social (inter)action, and cultural production" (Leppänen \& Häkkinen, 2012, p. 17). It is through such digital practices that the circulation and flow of ideas, discourses, and materials are accelerated and globally available resources are re-purposed and appropriated by individuals. 
The digital activities of multilingual users in connection with their identity work in these spaces hold a keen potential in the growing body of the scholarship. The research shows that multilingual speakers use semiotic resources including text, videos, photos, and hyperlinks for the manipulation and design of their profiles (e.g., Chen, 2013), and that both their personal and collective identities reflect local and global concerns (Thorne, Sauro, \& Smith, 2015). While identity formation and management of second language speakers in participatory spaces are explored in a number of studies (e.g., Klimanova \& Dembovskaya, 2013; Sharma, 2012), we have yet to see the research exploring individuals' employment of linguistic and multimodal resources in relation to their identity construction and management in SNS contexts. As Androutsopoulos (2013a) pointed out, the scholarship was more interested in the affordances of the technology-mediated communication forms rather than what people actually do with the options afforded by these platforms. The present study's goal was to illustrate how multilingual individuals used various tools and employ their linguistic repertoires to signal affiliation with different communities in a SNS context. For this purpose, the study examined the multimodal and multilingual social media practices of international graduate students, who experienced transcultural and transnational mobility abroad. It was aimed that the quantitative and qualitative analyses of the collected data exhibit how multilingual students maintain their legitimate status among home country groups while expanding identity roles through engagement with new host country communities within the same online space.

\section{LITERATURE REVIEW}

The advent of new forms of computer-mediated-communication (CMC) including SNSmediated technologies has brought academic attention to the participatory and superdiverse nature of these spaces. Among the areas explored were the communicative and performative activities of users in SNSs leading to construction of complex and dynamic identities. This body of research indicates that the mediated nature of SNS platforms offer a virtual space for individuals to perform who they are (Bouvier, 2012), carefully create an image for themselves, and craft their digital identities (Maghrabi, Oakley, \& Nemati, 2014). Going beyond whether identities can be constructed in SNS, the up-to-date literature (e.g., Chen, 2013; Klimanova \& Dembovskaya, 2013) began to deal with the function of SNSs in the (re)construction and transformation of learner identities.

Investigation of the question how SNSs provide an environment for the construction of multilingual users' identities reveals that social, technical and multimodal characteristics of these spaces are paramount in identity work. A number of research studies exploring identity management online (e.g., Greenhow \& Robelia, 2009; Young, 2013) show that SNSs offer multimodal tools for individuals to present themselves through verbal and non-verbal means. The purposeful choices of language in status updates and comment sections (i.e., multilingual repertoires), shared images, links, drawings, the use of abbreviations and emoticons in SNSs also reflect the agency of the users and project certain identities (Jonsson \& Muhonen, 2014). Mills (2011) noted that discursive practices of learners had assisted them in the process of adopting new, dynamic, and shifting identities through language. Similarly, DePew (2011) argued that learners harness their multilingual repertoire while presenting themselves to different audiences. Klimanova and Dembovskaya (2013) defined L2 speaker identity as a "dynamic identity-in-the-making" (p. 82) and observed that Russian learners redefine their identities by contesting presuppositions or developing further attributes throughout the conversation with project partners. Sharma's (2012) analysis in informal contexts, on the other 
hand, exhibited how Nepali youth employed English as an L2, and other semiotic resources in order to construct cosmopolitan personae.

The analysis of digital practices in SNSs necessitated a reconsideration of the ways scholars approached the data as well. In response to the theoretical and methodological needs for analyzing complex multilingual practices in online contexts, Androutsopoulos (2013a) introduced Networked Multilingualism, which requires researchers to take digital literacy repertoires, networked resources, and networked audiences into account when analyzing digital practices of individuals. This approach covers multilingual practices, which are shaped by interrelated processes of being networked (i.e., being connected to the users in one's network) and being in the network (i.e., embedded situation of an individual in the global web). Networked Multilingualism's promise comes from the fact that it not only prioritizes the multimodal and heterogonous materials, but also gives importance to the flows of these resources and how they are appropriated, entextualized, and translocalized. Furthermore, Networked Multilingualism highlights the importance of networked audiences for an understanding of how linguistic repertoires are used in various forms and genres in relation to the audiences in the network.

Further insights regarding the investigation of identity performances in digital spaces come from the strand of research investigating the phenomena of superdiversity in digital contexts. In line with post-structural perspectives on identity, Leppänen, Kytölä, Jousmäki, Peuronen, and Westinen (2013) conceptualized complex (dis)identification work in social media as "a dynamic and multi-faceted process involving affinity, alignment, emotional attachment and ideological notions of togetherness" (p. 5), while Blommaert and Varis (2011) identified a critical concept in the analysis of identity work in transnational participatory spaces such as SNSs. Calling the benchmark "enoughness" (p. 4), they argued that "one has to "have' enough of the emblematic features in order to be ratified as an authentic member of an identity category" (p. 4). In addition to these arguments, Leppänen et al. (2013) joined a number of other researchers by emphasizing the vitality of terms like re-entextualization (i.e., repositioning a discourse material as a meaningful element in a new context) and resemiotization (i.e., rearticulation of meaning across multiple modes and modalities) as multimodal resources for identity performances. They suggested the use of these terms as analytical notions for understanding complex social media practices.

Overall, since digital media enables the use of semiotic resources and the circulation of them across networks, the functions of such re-contextualization practices, multimodal resources and multilingual repertoires for displaying and managing various aspects of identities deserve special attention. Online identity formation and management of international graduate students (IGSs) are particularly worthwhile examining since they live beyond national borders and are exposed to diverse social and cultural traditions. Individuals like IGSs "develop meaningful ties to more than one home country, blurring the congruence of social space and geographical space" (Wei \& Hua, 2013, p. 517). Similar to immigrants and refugees, they try to deal with the complexities of belonging both their home and host countries ('here' and 'there') simultaneously (Suárez-Orozco, 2001). An examination of these students' digital practices may reveal how these individuals align or (dis)identify themselves within a complex and heterogeneous network. Besides, they often speak English as a second or foreign language, therefore they have relatively rich multilingual repertoires. As IGSs experience transcultural mobility, the audiences in these individuals' networks are also likely to be diverse. Investigating IGSs' language practices, the ways their language choices function in addressing 
a set of audiences and signaling their affiliation with various groups can maximize the understanding of the role of individual repertoires in online superdiverse spaces.

Building on earlier research and guided by the insights of Networked Multilingualism and post-structural identity views, this study aimed to investigate linguistic and semiotic resources deployed by IGSs in developing particular trajectories of identification at a moment marked by mobility in their lives. For this purpose, the present study's goal was to understand to what extent multilingual students used multimodal and multilingual resources in SNSs and what kinds of linguistic repertoires they employed for (dis)identification purposes.

\section{RESEARCH METHOD}

This paper is part of a larger project which included 90 international graduate students across the United States (Solmaz, 2015, 2017). For the current part of the project, I selected 13 participants pursuing graduate degrees at a higher education institute in southwestern United States (see Table 1). I recruited the students through an online survey conducted as part of the larger project, in which they indicated to volunteer for the study. This group of students was limited to a single higher education institute since I frequently needed to meet participants and conducted face-to-face semi-structured interviews with them. However, in order to draw a comprehensive picture, I prioritized volunteers with various backgrounds such as age, gender, pursued degree, academic program, country of origin, and length of stay in the United States. All of the participants had membership across multiple social networking site platforms.

Table 1. Participant's profile information.

\begin{tabular}{|c|c|c|c|c|c|}
\hline Name & Sex & $\begin{array}{l}\text { Home } \\
\text { country }\end{array}$ & Field of study & $\begin{array}{c}\text { Pursued } \\
\text { degree }\end{array}$ & $\begin{array}{l}\text { Length of } \\
\text { Stay (yrs) }\end{array}$ \\
\hline Ahmed & $\mathrm{M}$ & Egypt & Applied Linguistics & $\mathrm{PhD}$ & $4+$ \\
\hline Carlos & M & Costa Rica & Linguistics & $\mathrm{PhD}$ & $4+$ \\
\hline Elif & $\mathrm{F}$ & Turkey & Special Education & $\mathrm{PhD}$ & $4+$ \\
\hline Estella & $\mathrm{F}$ & Spain & Hispanic Studies & Masters & $1+$ \\
\hline Gabriel & M & Colombia & Applied Linguistics & $\mathrm{PhD}$ & $2+$ \\
\hline Gloria & $\mathrm{F}$ & Mexico & Arid Lands Resources Sciences & $\mathrm{PhD}$ & $3+$ \\
\hline Isabel & $\mathrm{F}$ & Chile & Teaching and Teacher Education & $\mathrm{PhD}$ & $4+$ \\
\hline Nara & $\mathrm{F}$ & Indonesia & English as a Second Language & Masters & Less than 1 \\
\hline Nissa & $\mathrm{F}$ & Thailand & Applied Linguistics & $\mathrm{PhD}$ & $3+$ \\
\hline Thomas & M & Germany & Economics & $\mathrm{PhD}$ & $3+$ \\
\hline Wang & $\mathrm{F}$ & China & East Asian Studies & $\mathrm{PhD}$ & $2+$ \\
\hline Yeong & $\mathrm{F}$ & S. Korea & Communication & Masters & $1+$ \\
\hline Zahra & $\mathrm{F}$ & Pakistan & Applied Linguistics & $\mathrm{PhD}$ & $3+$ \\
\hline
\end{tabular}

Note. All names are pseudonyms to ensure participant's confidentiality.

I collected the data through semi-structured-interviews, and SNS data of the participants. The latter encompassed automatically-extracted and manually-collected data from participants' accounts. Therefore, the data was blended and consisted of both screen-based data (i.e., SNS data) and user-based data (i.e., interviews), which are considered as two main sites of data collection in new media sociolinguistics (Androutsopoulos, 2013b). I followed a cyclical and 
non-linear procedure through data collection in order to ensure a higher level of ethnographic engagement of the researcher.

Following the piloting process with two students resembling the target population, I contacted volunteers and requested them to share their Facebook data through Netvizz, a data collection and extraction tool developed for research purposes. I used these automaticallyextracted data for the purpose of visualizing network maps of participants through Social Network Analysis (SNA). The maps were created with Gephi Graph Visualization and Data Software. Although the research involving SNA often employs it for analytical purposes, this study used SNA as a data collection tool as well as the maps to elicit further information from the international graduate students. The use of maps during the interviews created a valuable opportunity to explore issues that might not have been discussed otherwise and allowed participants to talk more about topics such as audiences in their network, and the relationship between their linguistic choices and addressing various communities.

Apart from SNA, I quantitatively analyzed the social media data through the categorization of participants' posts according to linguistic and multimodal resource. I maintained 'language' as a coding category in the analysis of participants' multilingual repertoires. Since the participants had diverse linguistic backgrounds, I preferred coding categories such as first language (L1, self-identified native language of the participant), second language (L2, used for English) over labels such as Urdu, Chinese or German.

I supplemented the quantitative analysis with a fine-grained qualitative analysis of both interview transcripts and social media data, which covered materials shared/posted in a time period of at least 10 months and as much as 2 years. Drawing on Computer-Mediated Discourse Analysis (CMDA) (Herring, 2004) and its application to four different domains of language (structure, meaning, interaction, and social behavior), language-focused content analysis of the data was performed and a re-analysis of the data was run as the themes across interview and SNS data became prevalent. Emerging patterns such as ideas, interactions, and linguistic preferences were identified and organized into more coherent categories. Recurring themes across the data eventually formed major themes and a thematic organizational framework was created as a result of both etic (researcher-outsider) and emic (participantinsider) focus. Consistent reflection and recursive analysis allowed the study to be possibly more profound compared to a linear analysis process, which is seen as a separate step to be taken after data collection (Glesne, 2010)

\section{RESULTS}

This section encompasses findings of the analysis in two categories. While quantitative analysis of the participants' social media data presents a holistic view of participants' repertoires, both visualized network maps and the qualitative analysis of interviews and SNS data exhibit the role of participants' repertoires in their involvement in the participatory culture.

\section{Multilingual and Multimodal Repertoires of Participants}

The quantitative analysis of participants' linguistic choices included statistical counts of the participants' SNS data based on the emerging coding categories. The analysis included all the text-only status updates/posts as well as multimodal posts (i.e., photos, videos, web resources/links) which were accompanied by text. Since the coding was based on textual components and was conducted for the purpose of understanding linguistic practices, the 
participants' posts participants shared without text were not included in the quantitative analysis (see Table 2).

The analysis of the posts shows that linguistic practices of participants included the use of their first language (L1), second language (L2, English), multiple languages (L1 and L2 at the same time, ML) and translanguaging (TL) practices. The distribution of the language choices was not same for all the participants, and the numbers were widely varied. However, the overall result indicated that both L1 and L2 were almost equally present in online social networks, being $42.21 \%(n=799)$ and $42.70 \%(n=828)$ respectively. The co-presence of L1 and L2 was found in $12.53 \%(n=243)$ of the posts. Finally, there were instances of translanguaging practices, in which "meaning-making is not confined to the use of languages as discrete, enumerable, bounded sets of linguistic resources" (Creese \& Blackledge, 2015, p. 21). These practices, however, were present only in $3.56 \%(n=69)$ of the SNS posts.

Table 2. Participants' language choices for text-only and multimodal posts on Facebook.

\begin{tabular}{|c|c|c|c|c|c|c|}
\hline \multirow[t]{2}{*}{ Pseudonym } & \multirow[t]{2}{*}{ Posts } & \multicolumn{5}{|c|}{ Language Choices } \\
\hline & & L1 & L2 & ML & TL & Total \\
\hline \multirow[t]{2}{*}{ Ahmed } & Text-only & 1 & 4 & 1 & 2 & 8 \\
\hline & Multimodal & 31 & 33 & 1 & 4 & 69 \\
\hline \multirow{2}{*}{ Carlos } & Text-only & 4 & 2 & 3 & 0 & 9 \\
\hline & Multimodal & 6 & 2 & 11 & 2 & 21 \\
\hline \multirow[t]{2}{*}{ Gabriel } & Text-only & 4 & 1 & 3 & 1 & 9 \\
\hline & Multimodal & 14 & 9 & 7 & 0 & 30 \\
\hline \multirow[t]{2}{*}{ Gloria } & Text-only & 7 & 2 & 2 & 0 & 11 \\
\hline & Multimodal & 23 & 5 & 9 & 0 & 37 \\
\hline \multirow[t]{2}{*}{ Elif } & Text-only & 56 & 12 & 5 & 2 & 75 \\
\hline & Multimodal & 107 & 38 & 3 & 5 & 153 \\
\hline \multirow[t]{2}{*}{ Estella } & Text-only & 13 & 5 & 5 & 3 & 26 \\
\hline & Multimodal & 40 & 22 & 5 & 2 & 69 \\
\hline \multirow[t]{2}{*}{ Isabel } & Text-only & 56 & 26 & 11 & 4 & 97 \\
\hline & Multimodal & 109 & 34 & 13 & 2 & 158 \\
\hline \multirow[t]{2}{*}{ Nara } & Text-only & 102 & 186 & 39 & 9 & 336 \\
\hline & Multimodal & 29 & 104 & 22 & 2 & 157 \\
\hline \multirow[t]{2}{*}{ Nissa } & Text-only & 14 & 4 & 7 & 3 & 28 \\
\hline & Multimodal & 88 & 104 & 41 & 0 & 233 \\
\hline \multirow{2}{*}{ Thomas } & Text-only & 0 & 7 & 0 & 0 & 7 \\
\hline & Multimodal & 1 & 22 & 2 & 1 & 26 \\
\hline \multirow{2}{*}{ Wang } & Text-only & 44 & 56 & 13 & 21 & 134 \\
\hline & Multimodal & 26 & 45 & 6 & 0 & 77 \\
\hline \multirow[t]{2}{*}{ Yeong } & Text-only & 14 & 3 & 6 & 0 & 23 \\
\hline & Multimodal & 6 & 19 & 21 & 1 & 47 \\
\hline \multirow[t]{5}{*}{ Zahra } & Text-only & 4 & 33 & 6 & 1 & 44 \\
\hline & Multimodal & 0 & 50 & 1 & 4 & 55 \\
\hline & Average & 30.73 & 31.8 & 9.34 & 2.65 & 74.5 \\
\hline & Total number & 799 & 828 & 243 & 69 & 1,939 \\
\hline & Percentage & $41.21 \%$ & $42.70 \%$ & $12.53 \%$ & $3.56 \%$ & $100 \%$ \\
\hline
\end{tabular}

Note. L1: First Language; L2: English; ML: Multilingual, TL: Translanguaging 
In order to display the role of various digital media and multilingual resources employed and repurposed by the participants, I analyzed the difference between text-only and multimodal posts of participants and differences across language selections as well (see Table 3).

Table 3. Percentages and numbers of participants' multimodal and text-only posts on Facebook.

\begin{tabular}{cccc}
\hline Participant & Multimodal \%. (Number) & Text-only \%. (Number) & Ratio \\
\hline Nara & $32(157)$ & $68(336)$ & 0.47 \\
Wang & $36(77)$ & $64(134)$ & 0.56 \\
Zahra & $56(55)$ & $44(44)$ & 1.28 \\
Isabel & $62(158)$ & $38(97)$ & 1.63 \\
Elif & $67(153)$ & $33(75)$ & 2.03 \\
Yeong & $67(47)$ & $33(23)$ & 2.03 \\
Carlos & $70(21)$ & $30(9)$ & 2.33 \\
Estella & $73(69)$ & $27(26)$ & 2.7 \\
Gabriel & $77(30)$ & $23(9)$ & 3.35 \\
Gloria & $77(37)$ & $23(11)$ & 3.35 \\
Thomas & $79(26)$ & $21(7)$ & 3.76 \\
Nissa & $89(233)$ & $11(28)$ & 8.1 \\
Ahmed & $90(69)$ & $10(8)$ & 9 \\
\hline Total & $67 \%(1132)$ & $33 \%(807)$ & 3.12 \\
\hline
\end{tabular}

Table 4. Percentages and numbers of participants' posts in L1 and L2 on Facebook

\begin{tabular}{|c|c|c|c|}
\hline Participant & L1 \% (n) & $\mathrm{L} 2 \%(n)$ & Ratio \\
\hline Gloria & $81(30)$ & $19(7)$ & 4.26 \\
\hline Elif & 77 (163) & $23(50)$ & 3.35 \\
\hline Isabel & $73(165)$ & $27(60)$ & 2.7 \\
\hline Carlos & $71(10)$ & $29(4)$ & 2.45 \\
\hline Estella & $66(53)$ & $34(27)$ & 1.94 \\
\hline Gabriel & $64(18)$ & $36(10)$ & 1.78 \\
\hline Nissa & 49 (102) & $51(108)$ & 0.96 \\
\hline Yeong & $48(20)$ & $52(22)$ & 0.9 \\
\hline Ahmed & $46(32)$ & $54(37)$ & 0.85 \\
\hline Wang & $41(70)$ & $59(101)$ & 0.7 \\
\hline Nara & $31(131)$ & $69(290)$ & 0.45 \\
\hline Zahra & $5(4)$ & $95(83)$ & 0.05 \\
\hline Thomas & $3(1)$ & $97(29)$ & 0.03 \\
\hline $\begin{array}{r}\text { Total } \\
\end{array}$ & $67 \%(799)$ & $33 \%(828)$ & 1.5 \\
\hline
\end{tabular}


According to Table 3, IGSs frequently employ multimodal resources when they share entextualized posts as the percentage of their multimodal posts was about twice that of textonly posts. Regardless of the personal motives, IGSs used multimodal means for various purposes including reconstruction of social and cultural contexts, managing relationships within a networked audience, and meaning-making performances.

I demonstrate the percentages and number of participants' L1 and L2 posts on Table 4 above. Based on the table, the presence of L1 in SNSs of IGSs was more apparent than L2 even though there were considerable differences between participants. Interestingly, all the participants who use L1 more than L2 in their network speak Spanish as their first language (Gloria, Elif, Isabel, Carlos, Estella, Gabriel) with the exception of a Turkish speaker (Elif). While L2 was present as much as L1 in the networks of Nissa, Yeong, and Ahmed, L1 was more dominant in the SNSs of Wang, Nara, and specifically Zahra and Thomas.

Based on these results and interviews with the individuals, I draw a connection between the language choices of participants in SNS with the dominance of particular language(s) spoken by audiences in their networks and the active presence of particular communities. For instance, L1 speaking audiences in the networks of Gloria, Estella, and Elif, and L2 speaking audiences in the networks of Zahra, Wang and Thomas were self-reportedly higher in terms of number (or were more active as in Thomas' case). The language choices of Isabel and Gabriel, whose family members in their home countries expect them to share multimodal updates, were influenced by these expectations to some extent and this contributed to the dominance of L1 in their networks since they preferred using L1 in such cases. The distribution of languages in Nissa and Ahmed's networks were close to the overall distribution of L1 and L2 speaking audiences in their networks. While the same thing might be said for Nara's network, her L2 was the dominant preference in her network. It might be because of Nara's purposeful choice of emphasizing a particular English-speaking cosmopolitan persona that she wanted to display for her students. The case of Yeong was particularly interesting as she had a dominant Koreanspeaking network (see Figure 5). Considering the higher number of multiple language use by Yeong, accommodating audiences in her network was important for her.

In order to illustrate the employment of multimodal and multilingual resources in the projection of identity performances visually, I created the figure above with the statistical ratio of multimodal posts to text-only updates and the ratio of L2 use to L1 use (see Figure 1). The intersection of horizontal and vertical green lines below are aligned with the equivalent practice of both text-only and multimodal posts, and L2 and L1 use. While the horizontal location of an individual shows their L2:L1 ratio, their vertical location shows their ratio rate of multimodal posts to text-only updates. Multimodal posts were strongly preferred over text-only posts by almost all of the individuals, and the language choices were more varied. Based on the analysis, I argue that certain preferences and interests affect the resource choices in SNS. For instance, Wang and Nara, the only individuals with more text-only updates than multimodal posts, constantly adopted a narrative style in their status updates and often reflected on their daily life experiences. On the other hand, Ahmed, who has the highest non-linguistic resource use among the participants, often shared content through other websites or via Facebook pages and his friends. The other highly multimodal user, Nissa, specifically mentioned that she was a visual person and often used semiotic content in her posts. In addition to these, Nara's interest in creative writing and Nissa's interest for photography also seemed to have a role in the former's preference for text-only and the latter's preference for multimodal posts. This initial finding and interpretation require further testing especially for a better understanding of what kinds of factors affect the semiotic resource use of individuals in SNSs. 


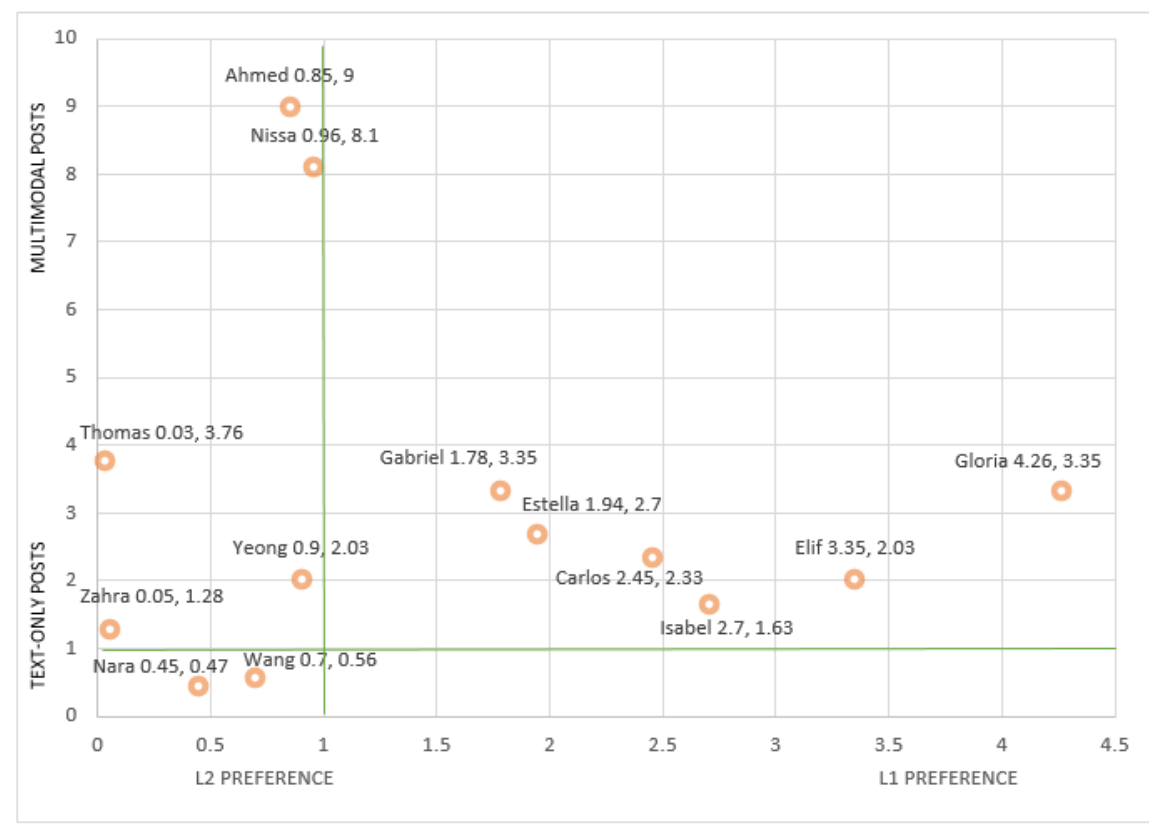

Figure 1. Ratio chart of participants' text-only and multimodal posts, and L2 and L1 posts.

Despite their different choices, it was clear that language practices of IGSs in SNS contexts were multilingual in nature and these individuals used resources from a multitude of linguistic repertoires. While L1 and L2 use were dominant in their networks, the simultaneous use of multiple languages (often L1 and L2 together) and translanguaging practices occurred as well.

\section{Multilingual Practices of Participants}

In this section, I present a more detailed picture of multilingual repertoires and practices of participants in SNS spaces through SNS data samples, visualized maps, and interview excerpts for the linguistic categories given above.

\section{The Use of First Language (L1)}

The use of first language (L1) by IGSs was among the most common linguistic practice. The results indicated that $\mathrm{L} 1$ use was closely related to the presence of relevant audiences as well as other factors such as the convenience of using native language and being more competent in that particular language. Overall, L1 use in SNSs offered a discursive way for IGSs to project certain aspects of their local identities.

The presence of a networked audience in an individual's SNS account had impact on participants' linguistic practices. Isabel reported that she used only Spanish while thinking about Chile or something related to it. She further added that if she wanted to post something that addressed her family [in Chile], she would do it in Spanish. Similarly, Gabriel shared his adventures with his family often in Spanish or both Spanish and English. By employing the linguistic medium, both Isabel and Gabriel positioned themselves as Spanish/multilingual parents who communicated with their family in Latin America. 
In line with the cases above, Gloria mentioned the importance of knowing her audience and how that led to certain linguistic choices:

I actually have a lot of friends who speak Spanish, so it is kind of easier to that if
you are in this region [Southern Arizona]. I have at least 10 American friends who
speak Spanish as well. So, they are like the bridges of information. I can share
those [resources] with them and I am pretty sure they will talk about it to their
American friends who do not speak Spanish. - Gloria

Audience awareness led Gloria to employ an audience-specific linguistic practice and assisted her to reach an audience beyond her network. Similarly, Estella engaged in Spanish language practices partly thanks to her network in the United States, a majority of whom consisted of her Spanish-speaking colleagues. I observed that the audience in participants' home countries, the new network formed in the United States, and the context of being in a Spanish department in a bilingual region might lead to the L1 use.

Some participants also reported that their L1 choices were sometimes affected by their mood, the context of the post, and the purpose of developing empathy. Yeong mentioned that she could elaborate more in her native language and talk about her deeper emotions and thoughts better. She also noted that people in Korea would empathize with her. Nissa, who shared a post related to her frustration about the statistics class, explained her use of Thai by saying:

\section{It [the post] is mostly about complaining and I like to complain in Thai! I feel like my friends in Thailand would show more empathy towards me because all of them would have to go through this [in Thailand]. -Nissa}

Linguistic repertoires of the participants displayed diversity within their native languages in some cases. For example, Gloria made use of her Sonoran dialect of Spanish, while Estella, a native of Canary Islands, used a variety of their hometown Spanish in some cases. For instance, Estella adopted an informal Spanish, which her mother often used, such as using $\mathrm{Pa}$ ' casa instead of a more common Para casa (to go home). Even though her post was in Spanish, as she made use of SNS feature offering its users to specify their current locations and arrival targets at the time of mobility, her status update became multilingual in nature.

Gloria also mentioned that she made lexical choices which would be understood only by her friends in northern Mexico. By noting that a lot of her posts had "that kind of flavor," she pointed out that she often used Sonoran dialect to talk about personal things. She reported a story about little girl who moved next to her apartment with her Chilean family. The little girl did not have any friends in her new home. Upon witnessing this, Gloria shared a photo with the text "No hay mas chamacos para jugar" (There are no more children to play). She identified the word 'chamaco' as "very Northern" during the interview. She stated that Sonoran people would understand that this situation was personal and emotional to her through this way. This is an example in which one can see the power behind the use of a single lexical item from the linguistic repertoire of home country. The use of a local variety not only helped Gloria convey her emotions in a deeper way, it also became a rich medium for her to index her Sonoran identity. It is reported that even a single utterance from a local variety of first language can index ethnic identity of speakers while facilitating the communication with local interlocutors (Duff, 2015). For both cases, such local varieties of L1 often signal specific affiliations with particular groups among home country audiences. 


\section{The Use of Second Language (L2 / English)}

Participants exhibited their second language (L2) repertoires as much as their L1s. Positioning themselves as competent users of English, their written discourses in L2 allowed them to index global aspects of their identities in a superdiverse context. Similar to native language practices, the role of audience in the selection of L2 was important. Since the participants had been studying in the United States for a while, their networked audience included speakers of English from their host country. Besides, IGSs reported that linguistic repertoires of their audiences were multilingual.

A common way of using L2 included posts addressing particular community clusters where English was dominantly used. For instance, Isabel, who worked as a graduate assistant, said that she would use English when she talked about work because she knew who would read it. Similarly, participants often chose English over their native language when they shared graduate school or professional work-related updates (see Figure 2). Communicating in English has become a valuable cultural capital in their identification as graduate students. In line with these, another participant (Ahmed) reported that most of the things he shared in English at some point served the purpose of raising awareness of his American audience.
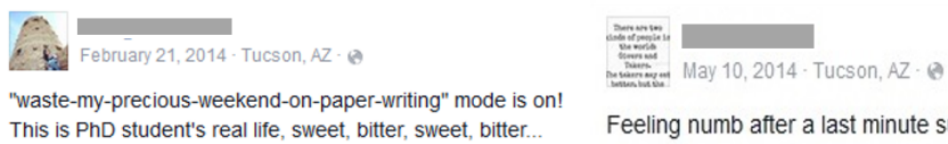

Feeling numb after a last minute submission :: hehe

Figure 2. Wang (left) and Zahra's (right) graduate school related status updates in English.

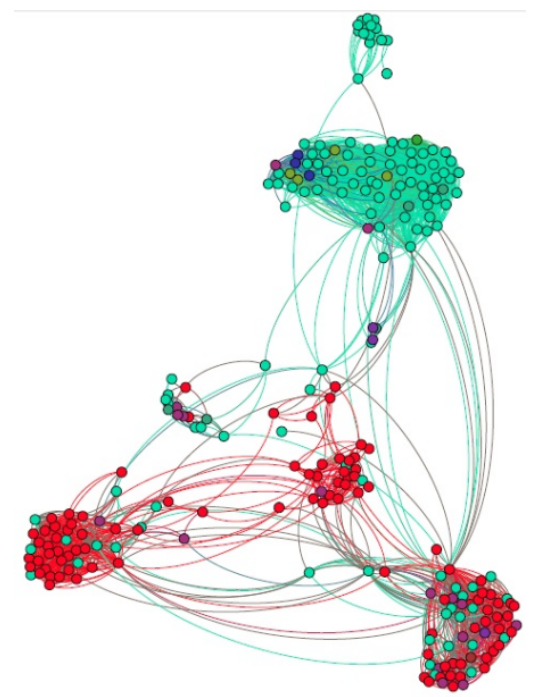

Figure 3. Thomas' visualized Facebook network map by language of interface [Red: German, Green: English]

The analysis of social networking data revealed that the presence of a community cluster in a network did not automatically translate to addressing of that community's linguistic repertoire. For instance, Thomas' visualized map showed that he had a number of community 
clusters consisting of people who used Facebook in English and/or German (see Figure 3). While nodes in red represent German users in Thomas' network, green-colored nodes stand for English users. Despite the distributed colors and relatively good presence of German speakers in Thomas' map, the quantitative analysis revealed that Thomas' entextualized posts in German were limited. During the interview, Thomas explained the situation:

I think the people from Germany that I am friends with on Facebook, a lot of people are more acquaintances, like people I know from high school, people I know from undergrad, people that I do not keep close contact anymore, and then those people with whom I do keep close contact with, I don't usually post that on Facebook. - Thomas

Thomas also revealed that one of the reasons he chose English over German was his perception of English as being comparatively more inclusive. He said that the choice of English helped him address all the people who did not speak German. He added that his network included more bilingual speakers whose native language was German compared to the other way around. Thomas concluded his ideas about using English on Facebook by saying: "[In the United States] I think in English, I dream in English, so why not use Facebook in English?" (Interview with Thomas). In line with this perspective, Nara shared her opinion on the inclusiveness of English as a medium of linguistic practice on Facebook: "If I want my post to be understood by more people, I use English basically. When I am not concerned about if all people will understand or not, I use any language I want" (Nara).

Overall, the results indicated that L2 use was a common linguistic practice for IGSs to effectively take part in the participatory SNSs, and IGSs employed it for various purposes and in different quantities.

\section{The Use of L1 and L2, \& Translanguaging}

The participants used multilingual resources in their digital activities in a number of ways. For instance, they used their L1 and L2 together as separate entities and both languages signified the same idea. They sometimes posted about the same topic in two different languages in a single status update but they elaborated more in one language over the other. Participants occasionally used more than one linguistic variety in a single status update, which demonstrated their multilingual abilities in a written context.

An example of the use of L1 and L2 together comes from Gabriel, who updated his status in Spanish and English after receiving birthday wishes from people in his network. Gabriel's multilingual status update, which started with Spanish text, was followed by the direct translation of his message in Spanish. Taking the languages used in birthday wishes and two dominant languages used by his networked audience, Gabriel thanked his friends in both Spanish and English.

Unlike the example given above, some participants' updates included two languages which conveyed a similar message with different lexical choices across the languages. The majority of Yeong's posts in which Korean and English were used consistently included such examples (see Figure 4).

Finally, I'm leaving Tucson soon and will arrive in Incheon airport Wednesday night (Korean time) $\odot$ wow Can't wait to be there! I will be there for a month which is not a short period of time but can actually feel extremely short, I hope to 
enjoy the time there at most and see all the people I missed and eat all the food that I missed! See y'all soon! (English translation of the Korean text in Figure 4)

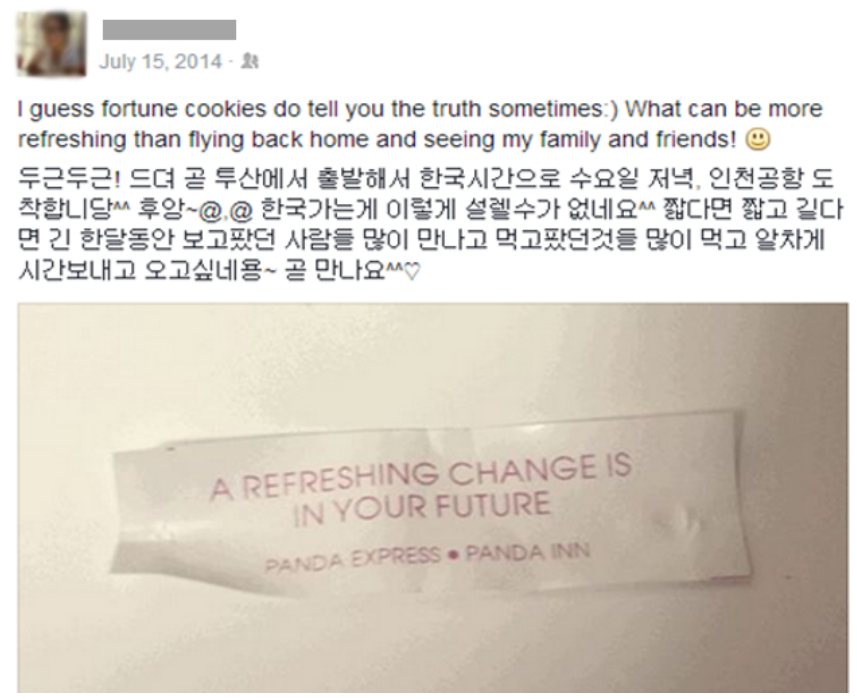

Figure 4. Yeong's update about her visit to Korea.

It is clear from the Korean text that she was aware of the linguistic backgrounds of her audience and aimed to address them appropriately. It is interesting to note that Yeong did not refer to the fortune cookie when she wrote in Korean. She reported that the fortune cookie was not a popular concept in South Korea and she did not need to mention it. However, she added that the fortune cookie in this post helped her start to talk about her home trip.

Presence of Korean speakers in Yeong's network was comparatively heavy (see Figure 5). In terms of the use of English and Korean together in Yeong's posts despite of a heavier Korean audience presence, Yeong noted that she wanted to include as many people as possible in her network. She further specified that her posts including both Korean and English were not exact translations although the topic was same. She reported that she knew what people in Korea would empathize more with and further added by expressing "I guess I know how to address them. It is usually to make them feel that I am still Korean" (Interview with Yeong). Marwick and boyd (2011) noted that people who could not handle multiplicity of audiences in online networks experienced 'context collapse' as they experience challenges in addressing them appropriately. By managing potential tensions between her Korean-only and non-Korean speaking audiences, as a multilingual speaker, Yeong not only chose different languages depending on the context but also talked about a particular sharing "differently when talking to different audiences" (boyd, 2011, p. 51). Management of an online performance to address different audiences through language practices within the same unit (i.e., a post) is an important finding as there is no empirical analysis of discourse in the literature (Androutsopoulos, 2014). 


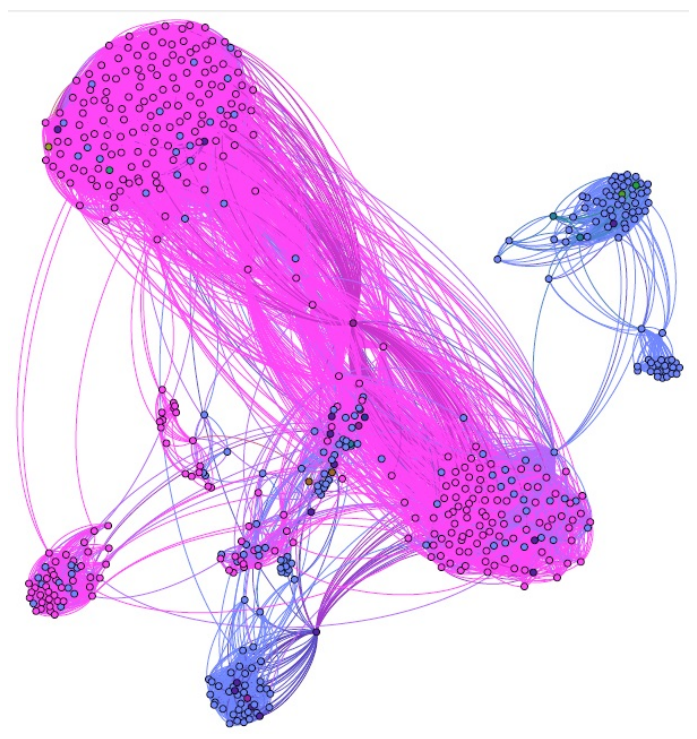

Figure 5. Yeong's visualized Facebook network map by interface language [Pink: Korean, Blue: English].

Participants switched between linguistic varieties in the course of a single sentence within a status update as well. Zahra posted a video which was re-entextualized with the use of Urdu and English languages in the same sentence. She wrote the text below (English translations of Urdu texts are given in brackets): "PS: Before my friends ask me, "Kya hua" [what happened], I wish to clarify dil wil nahi toota, gaana acha hai :p [my heart did not break, the song is good]."

When asked about this post, Zahra mentioned that the song was about a lady whose heart was broken by her lover. She noted that she loved the song and the intended audience of this post was her Pakistani colleagues and friends who lived in the United States. In this way, Zahra moved across her multilingual repertoire to address a particular community cluster in her network and positioned herself as a multilingual citizen who could skillfully make switches between different linguistic varieties and form her own creation. Alim (2009) called such translanguaging practices as an action of "agentive languaging" (p. 123) and pointed that such practices describe globalization best.

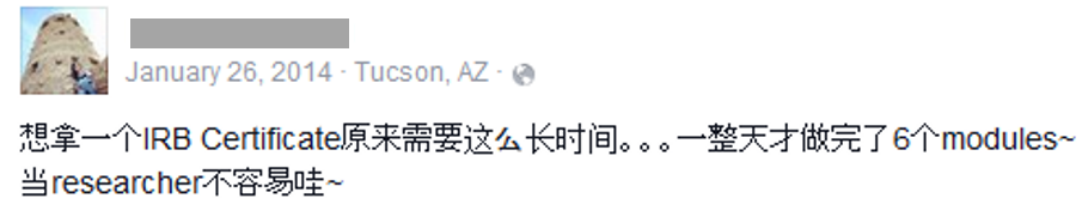

Figure 6. Wang's status update displaying her translanguaging practice.

In another case, Wang shared the post in Figure 6, which was translated into English below (English words used in her post underlined): "It turns out that I have to spend such a 
long time to get an IRB certificate. spent a whole day for just $6 \underline{\text { modules }} \sim$ it's not easy to be a researcher."

Wang, who sometimes integrated her English lexical choices into Chinese updates, used English words in the post regarding her experience with the required procedures of the Institutional Review Board (IRB). When asked about her selections, she noted that words like $I R B$ certificate and modules did not come to her mind at the time of posting. She added that she learned those words in English and that possibly affected her choice. She further expressed that the use of words like IRB certificate and modules in this particular sentence and their connection to the word researcher might have triggered her choices. As in Wang's case, IGSs' multilingual practices in the form of translanguaging could be related to factors other than one's networked audience. Regardless of the factors, such flexible and seamless shifts are claimed to develop and transform "speakers' skills, knowledge, experience, attitudes, and beliefs; thus creating a new identity for the multilingual speaker" (Li \& Zhu, 2013, p. 519). As seen in both cases above, participants engage in translanguaging, which has the potential to be an identity resource especially within transnational communities where individuals can underscore their in-betweenness and hybridity (Wei \& Hua, 2013).

Overall, the diverse multilingual repertoires of participants were reflected in their language choices. The presence and activity of audiences, self-presentation strategies, and some languages being perceived as lingua franca in a network sought to affect those choices. While L1 functioned as a strong marker of local identity, L2 allowed users to index global aspects of their identities. The presence of multiple languages at the same time projected relatively stronger cosmopolitan identification although translanguaging practices were quite limited. When L1 and L2 appeared together, they were often interpreted as independent entities as they were separately used even in single posts; thus exhibiting ordered practices and the transfer of normativity in daily life into online spaces.

\section{DISCUSSION AND CONCLUSIONS}

This study examined the multilingual and multimodal repertoires of international graduate students on Facebook, particularly focusing on how individuals employed semiotic and linguistic resources to index multiple aspects of their identity in social media. Findings suggested that language choices of IGSs were powerful means for these individuals to index certain affiliations with a wide variety of networked audiences. Following Blommaert and Varis (2011), who described identity discourses and practices as "discursive orientations towards sets of features that are viewed as emblematic of particular identities" (p. 3), language can be interpreted as a salient avenue for identification performances (Duff, 2015; Pavlenko \& Blackledge, 2004) and a major emblematic feature of particular identities. In light of this perspective, the current research showed that IGSs indexed identities by employing their linguistic repertoires in the form of L1, L2, simultaneous use of L1 and L2, and agentive languaging through translanguaging practices. It was sometimes in the form of L1 for maintaining membership among homeland audiences, or in L2 for establishing themselves as multilingual speakers of English, rather than as learners of English (Thorne et al., 2015). This finding is also in line with the work of Lee and Barton (2011), in which Flickr users were found to project local and global identities through multilingual literacy practices. However, the functions of the multimodal and multilingual choices are often not stable in online contexts. As the value of different languages are not equal in the linguistic marketplace (Pavlenko, 2000) and past research (Gu \& Tong, 2012) illustrated that mobility across spaces produced "varying 
index values for languages" (p. 501), the choice of a certain language resource and content, particularly of home country, not only indexed affiliation with home communities, but also functioned as a marker of identity in a space where non-home country audiences were present as well. Therefore, I suggest that researchers take this into account when analyzing digital practices of individuals experiencing transnational mobility.

The present study also showed that various factors affected participants' language choices including the presence of audiences, activity of audiences, and participants' shared linguistic repertoires with audiences. This study is in line with a number of studies, which consider networked audiences as an important factor in the participation and particularly employment of certain linguistic forms (Androutsopoulos, 2014; Lee \& Barton, 2011; Marwin \& boyd, 2011; Tagg \& Seargant, 2014). This paper added that the activeness of audiences and individual's strength of ties also played a role in engagement for some individuals as visually mapped through social network analysis. Thus, the presence of an audience group may not translate into the production of that group's linguistic repertoire all the time. The activeness of the audience and the shared interactional repertoires are also important factors for a successful interaction with heterogeneous, collapsed networked audiences.

An interesting finding of this study was the limited number of translanguaging practices employed by IGSs although it was reported that digital superdiverse contexts provided opportunities for emerging translanguaging practices (Schreiber, 2015) and group-specific linguistic productions among networked peers are encouraged (Androutsopoulos, 2013a; Seargant, Tagg, \& Ngampramuan, 2012; Sharma, 2012). Androutsopoulos (2014) argued that the production in a dialog in small clusters of networked peers was not same as orientation to a multitude of audiences. This argument was supported by this study as examples of translanguaging practices across these multilingual students' SNS data were rarely observed. I further argue that translanguaging practices require more overlapping repertoires and such productions are more likely to occur in smaller groups like earlier research explored, or affinity groups. The desire to extend linguistic normativity into digital spaces might also be a reason as the expected translanguaging among the young adult population of the study was less. This study clearly illustrated examples of what is called 'ordered practices' by showing the use of multiple languages as different entities and structures in the same post. Androutsopoulos (2014) also observed similar practices among his participants and called such posts "dual updates", while Cunliffe, Morris, Prys (2013) analyzed the use of Welsh by young bilinguals and considered such patterns of normativity as an extension of language behavior in face-to-face networks. By highlighting such ordered practices, this study provided evidence to the arguments of Higgins (2011):

Even as continued mobility and global flows produce new forms of language, culture, and social life in the new millennium, modern ideologies about standard varieties of language, national identities, and clear cut notions of ethnic and cultural identity will still continue to circulate. (p. 283)

Finally, the current study provided evidence for the management of online practices through language style. boyd (2011) argued that people manage their performances through the use of language (multilingual speakers choosing different language depending on context) and language style (users describing events differently when interacting with different audiences). Yeong's digital practices, for instance, were clearly not examples of translanguaging, but a reflective of designing for different audiences. She often designed her posts in Korean and English, yet unlike many other IGSs she talked about different aspects of the same event or 
situation in English and Korean languages when addressing different people in her network, thus managing her performance through language style.

Overall, I conclude that multilingual students frequently engage in linguistic and multimodal practices by mobilizing a great diversity of semiotic resources in their networks. The majority of participants' posts involved practices with a multimodal resource, and were often framed with a rich and varied set of linguistic resources, including L1, L2, and a combination and mixture of their L1 and L2. Multilingual students effectively utilized their repertoires to deal with the multiplicity of audiences and resources, and to build multiple identification performances. This finding clearly exemplified Canagarajah's (2007) arguments regarding people having multiple memberships in multilingual communities and holding "in tension their affiliation with local and global language groups as the situation demands" (p. 39) in an online context. I recommend that researchers test the findings and interpretations of this study for a comprehensive understanding of individuals' transnational identity work in social media. The use of visualized network maps for data collection purposes can assist researchers eliciting a richer data set through contact with participants. Future research incorporating social network analysis may be especially valuable for displaying connections and activities of individuals as well as groups, pages, and communities in online spaces.

\section{REFERENCES}

Alim, H. S. (2009). Translocal style communities: Hip Hop youth as cultural theorists of style, language, and globalization. Pragmatics, 19(1), 103-127.

Androutsopoulos, J. (2013a). Networked multilingualism: Some language practices on Facebook and their implications. International Journal of Bilingualism, 1-21.

Androputsopolous, J. (2013b). Online data collection. In C. Mallison, B. Childs, G. v. Herk (Eds.) Data collection in sociolinguistics: Methods and applications (pp. 236-250). New York, NY: Routledge.

Androutsopoulos, J. (2014). Languaging when contexts collapse: Audience design in social networking. Discourse, Context and Media, 4-5, 62-73.

Blommaert, J., \& Varis, P. (2011). Enough is enough: The heuristics of authenticity in superdiversity. Tilburg Papers in Culture Studies, paper 2.

Bouvier, G. (2012). How Facebook users select identity categories for self-presentation. Journal of Multicultural Discourses, 7(1), 37-57

Boyd, D. (2011). Social network sites as networked publics: affordances, dynamics, and implications. In: Papacharissi, Z. (Ed.), A networked self. Identity, community, and culture on social network sites (pp. 39-58). New York/London: Routledge.

Canagarajah, A. S. (2007). The ecology of global English. International Multilingual Research Journal, $1(2), 89-100$.

Chen, H. (2013). Identity practices of multilingual writers in social networking spaces. Language Learning \& Technology, 17(2), 143-170.

Cunliffe, D., Morris, D., \& Prys, C. (2013). Young bilinguals' language behaviour in Social Networking Sites: the use of Welsh on Facebook. Journal of Computer-Mediated Communication, 18(3), 339-361.

DePew, K. E. (2011). Social media at academia's periphery: Studying multilingual developmental writers' Facebook composing strategies. The Reading Matrix, 11(1), 54-75.

Duff, P. (2015). Transnationalism, multilingualism, and identity. Annual Review of Applied Linguistics, $35,57-80$.

Facebook (2017). Company Info, Newsroom. Retrieved from_http://newsroom.fb.com/company-info/

Glesne, C. (2010). Becoming qualitative researchers (4th ed.). Boston, MA: Pearson. 
Greenhow, G., \& Robelia, B. (2009). Informal learning and identity formation in online social networks. Learning, Media and Technology, 34(2), 119-140.

Gu, M. M., \& Tong, H. K. (2012). Space, scale and languages: Identity construction of cross-boundary students in a multilingual university in Hong Kong. Language and Education, 26(6), 501-515.

Herring, S. C. (2004). Computer-mediated discourse analysis: An approach to researching online communities. In S. A. Barab, R. Kling, \& J. H. Gray (Eds.), Designing for virtual communities in the service of learning (pp. 338-376). Cambridge: Cambridge University Press.

Higgins, C. (2011). Hybridizing spaces and the production of new identities. In C. Higgins (Ed.), Identity formation in globalizing contexts: Language learning in the new millenium (pp. 279-284). Berlin/Boston: Walter de Gruyter.

Jenkins, H. (2006). Convergence culture. New York, NY: New York University Press.

Jonsson, C., \& Muhonen, A. (2014). Multilingual repertoires and the relocalization of manga in digital media. Discourse, Context and Media, 4-5, 87-100.

Klimanova, L. \& Dembovskaya, S. (2013). L2 Identity, discourse, and social networking in Russian. Language Learning \& Technology, 17(1), 69-88.

Lee, C. K. M., \& Barton, D. (2011). Constructing glocal identities through multilingual writing practices on Flickr.com. International Multilingual Research Journal, 5(1), 39-59.

Leppänen, S., \& Häkkinen, A. (2012). Buffalaxed superdiversity: Representations of the other on Youtube. Diversities, 14(2), 17-33.

Leppänen, S., Kytölä, S., Jousmäki, H., Peuronen, S., \& Westinen, E. (2013). Entextualization and resemiotization as resources for (dis)identification in social media. Tilburg Papers in Culture Studies, paper 57.

Li, W., \& Zhu, H. (2013). Translanguaging identities and ideologies: Creating transnational space through flexible multilingual practices amongst Chinese university students in the UK. Applied Linguistics, 34(5), 516-535

Maghrabi, R. O., Oakley, R. L., \& Nemati, H. R. (2014). The impact of self-selected identity on productive or perverse social capital in social network sites. Computers in Human Behavior, 33, 367-371.

Marwick, A., \& boyd, D. (2011). "I tweet honestly, I tweet passionately": Twitter users, context collapse, and the imagined audience. New Media \& Society, 13, 96-113.

Mills, N. (2011). Situated learning through social networking communities: The development of joint enterprise, mutual engagement, and a shared repertoire. CALICO Journal, 28(2), 345-368.

Pavlenko, A. (2000). Access to linguistic resources: Key variable in second language learning. Estudios de Sociolinguistica, 1(2), 85-105.

Pavlenko, A., \& Blackledge, A. (2004). Introduction: New theoretical approaches to the study of negotiation of identities in multilingual contexts. In A. Pavlenko \& A. Blackledge (Eds.), Negotiation of identities in multilingual contexts (pp. 1-33). Great Britain: Multilingual Matters.

Schreiber, B. R. (2015). "I am what I am": Multilingual identity and digital translanguaging. Language Learning \& Technology, 19(3): 69-87.

Seargant, P., Tagg, C., \& Ngampramuan, W. (2012). Language choice and addressivity strategies in Thai-English social network interactions. Journal of Sociolinguistics, 16(4), 510-531.

Sharma, B. K. (2012). Beyond social networking: Performing global Englishes in Facebook by college youth in Nepal. Journal of Sociolinguistics, 16(4), 483-509.

Solmaz, O. (2015). Multilingual students' management of transnational identities in online participatory spaces (Unpublished doctoral dissertation). University of Arizona, Arizona.

Solmaz, O. (2017). Adapting new media literacies to participatory spaces: Social media literacy practices of multilingual students. Journal of Media Literacy Education, 9(1), 36-63.

Suárez-Orozco, M. (2001) Globalization, immigration, and education: The research agenda. Harvard Educational Review, 71(3), 345-366. 
Tagg, C., \& Seargant, P. (2014). Audience design and language choice in the construction and maintenance of translocal communities on social network sites. In P. Seargant \& C. Tagg (Eds.), The Language of Social Media (pp. 161-185). Basingstoke, Hampsire: Palgrave Macmillan.

Thorne, S., Sauro, S., \& Smith, B. (2015). Technologies, identities, and expressive activity. Annual Review of Applied Linguistics, 35, 215-233.

Wei, L., \& Hua, Z. (2013). Translanguaging identities and ideologies: Creating transnational space through flexible multilingual practices amongst Chinese university students in the UK. Applied Linguistics, 34(5), 516-535.

OSMAN SOLMAZ, PhD, works at the Department of Foreign Languages at Ziya Gokalp Faculty of Education, Dicle University in Turkey. Solmaz, a Fulbright alumnus, holds his M.A. and Ph.D. degrees from the interdisciplinary Second Language Acquisition and Teaching (SLAT) program at the University of Arizona in the USA. His research interests include international students, computer-assisted language learning and teaching, digital literacies, and teacher education. Email: osolmaz@dicle.edu.tr 